\title{
Pure motor demyelinating neuropathy: deterioration after steroid treatment and improvement with intravenous immunoglobulin
}

\author{
Michael Donaghy, K R Mills, S J Boniface, J Simmons, I Wright, Norman Gregson, \\ Jean Jacobs
}

\begin{abstract}
Within one month of starting oral prednisolone treatment weakness unexpectedly increased in four patients aged 34 to 75 years with purely motor forms of acquired chronic demyelinating neuropathy. By contrast, steroids produced the expected improvement in 11 other patients with symmetric sensorimotor chronic inflammatory demyelinating polyneuropathy. Two of the patients with purely motor demyelinating neuropathy were subsequently treated with high dose IVIg ( $0.4 \mathrm{~g} / \mathrm{kg} / \mathrm{day}$ for five days) with prompt improvements in strength measurements and motor nerve conduction. Thus IVIg seems to be the treatment of choice and steroids should be used with extreme caution, if at all, in patients with purely motor forms of acquired demyelinating polyneuropathy.
\end{abstract}

(F Neurol Neurosurg Psychiatry 1994;57:778-783)

This communication highlights unpredicted differences in the response to steroid treatment of different types of acquired chronic demyelinating neuropathy. These differences have important practical implications concerning the choice of appropriate treatment for patients with chronic inflammatory demyelinating sensorimotor neuropathy (CIDP), by contrast with those with purely motor demyelinating neuropathy or multifocal motor neuropathy (MFMN).

CIDP was first recognised as a treatable cause of neurological disability over 20 years ago after reports of steroid responsiveness, ${ }^{1-4}$ subsequently confirmed by a controlled trial. ${ }^{5}$ Azathioprine is often added to prednisolone, and occasionally causes improvement in patients who failed to respond to prednisolone alone.$^{67}$ Plasma exchange is effective in CIDP and is often used to initiate improvement in patients initially unresponsive to steroids. ${ }^{8}$ Two follow up studies of a total of 152 patients with CIDP suggest that between $85 \%$ and $95 \%$ of patients respond favourably to varying regimens of immunosuppressive treatment based on prednisolone, azathioprine, and plasma exchange. ${ }^{67}$
In recent years, various subtypes of MFMN have been characterised and associated with high titres of antiganglioside antibodies in $11 \%$ to $84 \%$ of cases depending on the clinical subtype. ${ }^{910}$ This motor neuropathy is most commonly multifocal and associated with motor nerve conduction block. Less often patients show symmetric weakness with diffusely slowed motor conduction velocities. These pure motor neuropathies have usually failed to respond to treatment with prednisolone. Indeed, progression of weakness seems to be common despite the use of high dose steroid treatment in MFMN. ${ }^{11}$ In one report, eight of nine patients improved with cyclophosphamide treatment ${ }^{11}$ but the prospect of serious side effects makes this an unattractive long term treatment for such a chronic disease. Improved strength has been reported recently after high dose IVIg treatment. ${ }^{12-15}$ In some cases IVIg or cyclophosphamide treatment had been started while the patient was already taking or had recently taken prednisolone and these reports of beneficial treatments must be seen in the light of the unexpected steroid induced weakness we report here.

\section{Methods}

PATIENT POPULATION

The study is based on retrospective analysis of 12 patients with CIDP and four with MFMN referred consecutively over a three year period to a peripheral neuropathy clinic run by one physician (MD). Diagnosis, decisions to treat, and monitoring of treatment were all undertaken by MD. Because the need for formal study of different neuropathy subgroups was not anticipated, there were no standard protocols for assessing the degree of weakness, the nature of the electrophysiological studies (performed by three different neurophysiologists), the necessity for sural nerve biopsy, or the estimation of serum ganglioside antibodies. Routine serum protein electrophoresis was undertaken on all patients and no paraproteinaemia was detected. Patients were diagnosed as having CIDP according to standard guidelines ${ }^{16}$; all had symmetric distributions of weakness, absence or reduced tendon reflexes, large fibre modality sensory loss, and 
absent or reduced sensory nerve action potentials (SAPs).

Pure MFMN was diagnosed on the following grounds:

(a) Purely motor features with normal sensation.

(b) $\operatorname{Arm}(\mathrm{s})$ affected at onset.

(c) Progression over more than two months.

(d) (Multi-) focal onset.

(e) Relative preservation of tendon reflexes even in affected limbs.

$(f)$ Motor nerve conduction block (defined as a proximal/distal CMAP amplitude ratio of $60 \%$ or less), absent or prolonged $\mathrm{F}$ waves, or motor conduction velocities (MCVs) reduced to $38 \mathrm{~m} / \mathrm{s}$ in the upper limb and $30 \mathrm{~m} / \mathrm{s}$ in the lower limb.

(g) Preserved SAPs or lack of demyelination or remyelination on the sural nerve biopsy.

SURAL NERVE BIOPSY

Subtotal sural nerve biopsy was undertaken at the ankle in the four patients with MFMN (1-4) to seek evidence of demyelination or remyelination. Plastic embedded semithin sections and teased fibre preparations were analysed morphometrically. ${ }^{17}$

\section{GANGLIOSIDE ANTIBODIES}

Frozen stored serum samples from the patients with MFMN (1-4) were analysed. GM1 and sialo GM1 antibodies were detected by enzyme linked immunosorbent assay (ELISA). ${ }^{18}$ This protocol has been validated in an international comparative study organised by $\mathrm{Dr}$ Zielasek (Würzburg). Further ganglioside antibody reactivity was detected by thin layer chromatography overlayer with a total ganglioside extract from human brain and cauda equina. ${ }^{18}$

\section{IMMUNOSUPPRESSANT REGIMEN}

All patients with CIDP or MFMN were treated with oral prednisolone $(60 \mathrm{mg} /$ daily) for the first two to four weeks, then with 45 $\mathrm{mg} /$ day until their neuropathic symptoms stabilised or improved. Treatment was then gradually converted to $25-45 \mathrm{mg}$ on alternate days for maintenance treatment. Azathioprine $(2.5 \mathrm{mg} / \mathrm{kg} /$ day $)$ was added in 10 of the 11 treated patients with CIDP, and in all four patients with purely motor neuropathy. Plasma exchange $(5 \times 4$ litres $)$ by continuous

MFMN patients: summary of investigations

\begin{tabular}{|c|c|c|c|c|c|c|c|c|}
\hline \multirow[b]{3}{*}{ Patient } & \multirow{2}{*}{\multicolumn{3}{|c|}{ Electrophysiology }} & \multicolumn{2}{|c|}{ Sural nerve biopsy } & \multirow{2}{*}{\multicolumn{3}{|c|}{$\begin{array}{l}\text { Ganglioside } \\
\text { antibodies }\end{array}$}} \\
\hline & & & & \multirow{2}{*}{$\begin{array}{l}\text { MF } \\
\text { density } \\
\left(\text { (mm }^{2}\right)\end{array}$} & \multirow{2}{*}{$\begin{array}{l}\text { (normal } \\
\text { range) }\end{array}$} & & & \\
\hline & $\begin{array}{l}M C V \\
(\mathrm{~m} / \mathrm{s})\end{array}$ & $\begin{array}{l}C B \\
(\%)\end{array}$ & $F(m s)$ & & & GM1 & $G D 1 b$ & GM2 \\
\hline 1 & $\begin{array}{l}15,32 \text { (arm) } \\
25 \text { (leg) }\end{array}$ & ND & 102 (arm) & 6100 & $(7500-1000)$ & + & + & - \\
\hline 2 & $\begin{array}{l}18,34 \text { (arm) } \\
29 \text { (leg) }\end{array}$ & 21 & Absent (arm) & 6729 & $(7500-8500)$ & - & - & - \\
\hline 3 & $\begin{array}{l}42,46 \text { (arm) } \\
36 \text { (leg) }\end{array}$ & $\begin{array}{l}59 \\
24\end{array}$ & $\begin{array}{l}\text { Absent (arm) } \\
64 \text { (leg) }\end{array}$ & 4038 & $(4000-7000)$ & + & + & + \\
\hline \multirow[t]{2}{*}{4} & $\begin{array}{c}15,16,30 \\
(\mathrm{arm})\end{array}$ & ND & 47 (arm) & 5901 & $(4000-7000)$ & - & - & - \\
\hline & 28,32 (leg) & ND & 75,76 (leg) & & & & & \\
\hline
\end{tabular}

$\mathrm{MCV}=$ Motor conduction velocity; $\mathrm{CB}=$ degree of conduction block (see methods) $\mathrm{F}=\mathrm{F}$ wave latency; $\mathrm{ND}=$ not done. flow was used in patients with CIDP taking more than two months to improve, and in the four patients with MFMN whose weakness seemed to increase after initiation of steroid treatment.

Patients 1 and 2 with MFMN were given IVIg $(0.4 \mathrm{~g} / \mathrm{kg} /$ day for five days) 33 months and 26 months respectively after they had last received any other immunomodulatory treatment (for example, steroids, plasma exchange, or cyclophosphamide). Before and after treatment, these two patients were assessed by myometry, a timed walk over a fixed distance, stamina tests of upper and lower limbs, and by electrophysiology.

\section{Results}

PATIENTS WITH SENSORIMOTOR CIDP

Patient profile

The 12 patients were aged 10 to 75 years. All had a mixed sensorimotor polyneuropathy; sensory ataxia was the predominant feature in one patient who also had mild weakness and electrophysiological evidence of motor nerve demyelination. Immunosuppressive treatment was started in 11 patients; one was not treated because of a combination of mild disability, diabetes mellitus, and low intelligence.

\section{Response to steroids}

After steroid treatment in the eight patients with CIDP aged 10 to 50 years, six regained normal or near normal limb muscle power within six months. One had mild pretreatment symptoms, barely sufficient to alter gait, and he improved only slightly. The patient with prominent sensory ataxia regained the ability to walk briskly without a stick, although some residual ataxia remained. Three older patients, aged 66 to 75 years, responded notably less promptly and completely to steroid treatment. The two patients in their eighth decade were initially unable to bear weight or walk but, after a combination of steroids, azathioprine, and plasma exchange, both made sustained improvements after four and eight months respectively, and eventually walked with walking frames.

None of the patients with CIDP deteriorated while taking steroids.

\section{PURE MOTOR DEMYELINATING NEUROPATHY (TABLE) \\ Patient profile}

The duration of symptoms in four male patients with MFMN ranged from five months to six years. Three patients $(1,3$, and 4) had a noticeably asymmetric pattern of muscle weakness. The weakness in patient 2 had started asymmetrically, but had later become symmetric. In three patients the arms were weaker than the legs, in two the weakness had commenced in one arm before spreading to predominate in the legs. None of the patients had sensory symptoms or signs. Patient 4 had undergone right ulnar nerve transposition four years earlier because of paraesthesia in the little finger; electrophysiological studies at that time had shown right 
median and ulnar motor conduction velocities of 15 and $16 \mathrm{~m} / \mathrm{s}$ although the patient did not recollect weakness.

The table summarises neurophysiological, nerve biopsy, and ganglioside antibody results. Neurophysiological studies showed that at least three nerves in each patient had one or more of the following features: motor conduction velocity less than $35 \mathrm{~m} / \mathrm{s}$ (arms) or $29 \mathrm{~m} / \mathrm{s}$ (legs), conduction block of more than $60 \%$, or prolonged $F$ wave latencies. The SAPs were normal in the two younger patients ( 1 aged 34 years; 2 aged 56 years). Neither the median nor the sural SAP were recordable in patient 4 (aged 75 years) despite a normal routine sensory examination. In patient 3 (aged 72 years), the radial SAP was $6 \mathrm{mV}$, the median $2 \mathrm{mV}$, and sensory examination was normal. Myelinated fibre densities in the sural nerve were within the normal range in patients 3 and 4, and were marginally below normal in the two younger patients ( 1 and 2 ). Teased fibres were examined in patients 1,2 , and 3 , and remyelinated segments were found in less than $1 \%$ of fibres; no segmental demyelination was observed. Two patients (1 and 3) had IgM GM1 antibody titres of $1 / 1000$ and $1 / 3000$ (90\% confidence interval for controls 1-180). On overlay both patients also showed IgM reactivity with GDIb, and one had weak reactivity with GM2. When run against human cauda equina gangliosides, three of the serum samples showed some reactivity with sulphated glucuronylparagloboside. ${ }^{19}$

\section{Response to steroids}

All four patients showed significant motor deterioration within four weeks of starting treatment with prednisolone $(60 \mathrm{mg} /$ daily). It was the striking nature of the deterioration that provoked this comparison with CIDP. In all four patients the rate of deterioration increased sharply and could not be regarded simply as an extension of the rather indolent rate of progression shown by each before treatment.

Patient 1 had previously had stable weakness of all four limbs. Within two weeks of prednisolone treatment he was unable to grip his car controls, bath taps or eating implements, and his signature deteriorated. $\mathrm{He}$ completely regained his presteroid motor function within four weeks of stopping steroids; no improvement had occurred with plasma exchange.

Patient 2 had previously had slowly progressive weakness of all four limbs. He rapidly lost the ability to walk within a month of starting steriods; within three months he could no longer roll over in bed or raise his legs or arms. Over five months the (right) ulnar nerve MCV fell from 34 to $12.5 \mathrm{~ms}$, the distal motor latency rose from 8.4 to $12 \mathrm{~ms}$. The median nerve MCV remained unchanged, but the distal motor latency extended from 10 to $19 \cdot 5$ ms. Courses of plasma exchange produced partial motor recovery lasting about two weeks. He slowly regained his presteroid strength when steroids were stopped after 10 months and cyclophosphamide $(100 \mathrm{mg}$ daily) was started for six months. Strength was then maintained after cyclosphosphamide was stopped.

Patient 3 became unable to walk, stand, shave, or feed himself within four weeks of starting prednisolone. Deterioration progressed, with temporary improvement after the first two of three courses of plasma exchange. He regained the ability to walk when cyclophosphamide (100 mg daily) was started, but this drug was stopped after Listeria meningitis and he subsequently died of overwhelming abdominal sepsis.

Patient 4 had slowly progressing asymmetric weakness of the arms, slight dysphagia, and leg unsteadiness. Within two weeks of starting prednisolone $(60 \mathrm{mg} /$ daily $)$ he was unable to stand, sit unsupported, or feed himself, and required nasogastric tube nutrition. Plasma exchange produced a slight temporary improvement in arm power. Six months after stopping steroids he had regained the ability to feed and swallow, and walk with a frame.

\section{Response to treatment with IVIg}

Patients 1 and 2 were treated with IVIg, and both showed an improvement in strength.

Patient 1 was treated with IVIg 33 months after stopping steroids at a time when he was able to walk $200 \mathrm{~m}$ wearing foot calipers, his hand and shoulders were weak, he was unable to write, and he had difficulty using cutlery and lifting a cup. For nearly three years he had needed help dressing and he had stopped driving because he was unable to hold the steering wheel. These deficits had been stable before declining slightly over six months. In December 1992 he was treated with IVIg. By the third day of IVIg treatment he considered that his arms were stronger. Over the next six weeks this improvement was maintained; he went swimming for the first time in two years and drove his car for the first time in three years; he was able to use a knife and fork and lift a cup; and shaved his chin for the first time in two years. He still had difficulty with fine finger movements including writing and manipulating buttons. His walking speed increased. There were large increases in the time he could abduct his shoulders against gravity and lift his heels off the bed against gravity (figure; $\mathrm{A}$ ).

In patient 1 the most pronounced change in serial measurements in nerve conduction made by a single observer was an increase in dispersion of the compound muscle action potential of the abductor digiti after stimulation at the elbow. Before treatment the compound muscle action potential from elbow stimulation had a duration of $23 \mathrm{~ms}$ with eight phases; after treatment the compound muscle action potential had a duration of $>80 \mathrm{~ms}$ with $>20$ phases. Ulnar motor conduction velocity from Erb's point to the elbow increased marginally from $24 \mathrm{~m} / \mathrm{s}$ to $31 \mathrm{~m} / \mathrm{s}$ but there was no consistent change in the velocity through the forearm. There was persistent EMG evidence of chronic denervation 


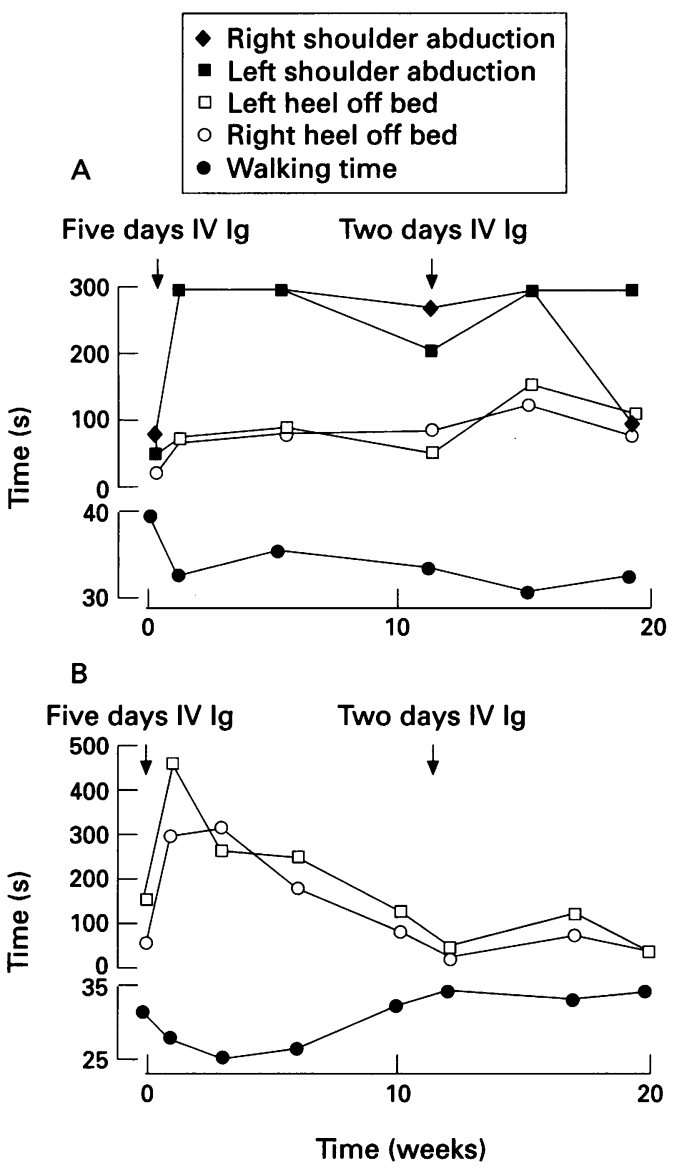

Walking time over a fixed distance and ability to hold limbs outstretched in relation to IVIg treatment $(0.4 \mathrm{~g} / \mathrm{kg} /$ day $)$. (A) Patient 1 ; (B) patient 2.

but sensory conduction remained normal and unchanged.

By 10 weeks after treatment with IVIg his strength had declined. $\mathrm{He}$ was once again finding difficulty in using eating implements, and shaving. His walking speed had slowed and the times for abducting his shoulders and raising his heels had declined, although these were still better than before treatment with IVIg. Patient 1 was then treated again, with a smaller dose of IVIg $(0.4 \mathrm{~g} / \mathrm{kg}$ for two days). As before he experienced a rapid increase in his strength, regained the ability to shave himself, and outstretched limb times improved once more (figure; A). This smaller dose was less beneficial and the patient reported a decline in strength within six weeks. A further small dose of IVIg ( $0.4 \mathrm{~g} / \mathrm{kg}$ for one day) produced slight improvement lasting only two to three weeks.

Patient 2 received IVIg 26 months after stopping cyclophosphamide. From October 1992 he noticed increased difficulty in walking, could not climb steps, and had to stop driving his car. In January 1993 he was admitted for IVIg treatment $(0.4 \mathrm{~g} / \mathrm{kg}$ day for five days). Seven days later his legs were stronger. Within two weeks his walking distance had improved and he required only one walking stick rather than two on inclines. He could climb steps again and restarted driving. His arms were stronger. Six weeks after treatment he considered that this improvement had been maintained. Myometry at one week showed improvement in the muscle groups tested except the first dorsal interosseous, although only three groups remained improved at six weeks. His walking speed was faster at one and six weeks and he could raise his legs against gravity for longer (figure; B).

In patient 2 the most pronounced early change in nerve conduction was an increase in the amplitude of the distal compound muscle action potentials. This rose from $0.8 \mathrm{mV}$ to $2.7 \mathrm{mV}$ for the right median nerve by the second day after treatment, retaining the normal biphasic waveform. The proximal compound muscle action potentials became more dispersed and polyphasic but $F$ waves were detectable in the right ulnar nerve at a minimum latency of $38 \mathrm{~ms}$. Motor conduction velocity was more variable, showing no consistent change in two out of three upper limb nerves and increasing from $18 \mathrm{~m} / \mathrm{s}$ to $42 \mathrm{~m} / \mathrm{s}$ after nine weeks in the right median nerve. Sensory conduction remained normal and unchanged.

From six to 12 weeks after treatment, patient 2 noticed a gradual return of his weakness. He could no longer confidently climb steps. He remained able to drive his car, and was still stronger than he was before treatment with IVIg. His walking time increased and the time he could keep his heels off the bed fell to pretreatment values (figure; B). A second, shorter course of IVIg $(0.4 \mathrm{~g} / \mathrm{kg}$ for two days) produced rapid improvement in strength and ability to walk up an incline or climb steps. The tests of his leg stamina improved, though not to as great an extent as with the initial treatment. This improvement was maintained for only five weeks, by which time his strength had declined; he was using two walking sticks and the times for lifting his heels off the bed had fallen to below the pretreatment values.

\section{Discussion}

This series of patients draws attention to the rapid deterioration in motor function that can be precipitated by high dose oral prednisolone $(60 \mathrm{mg} /$ day) in a motor, and often (multi-) focal, subgroup of chronic acquired demyelinating neuropathy. Such steroid induced deterioration only occurred in the four patients with purely motor symptomatology. It never followed steroid treatment in the 11 patients with symmetric sensorimotor CIDP. The prompt deterioration within four weeks of commencing prednisolone treatment, and the rapid improvement with plasma exchange or on stopping steroids, are all incompatible with the notion of steroid myopathy, which generally occurs after long term dosage with fluorinated steroids. As expected ${ }^{620}$ patients with sensorimotor CIDP improved with steroids; it was noteworthy that the younger patients improved more promptly and to a more satisfactory extent than the older patients. We regard the deterioration in MFMN as being due to steroid accelerated worsening of the underlying neuropathy. We did not undertake detailed neurophysiological 
monitoring of this unanticipated phenomenon. Its brisk responsiveness to plasma exchange, however, in patient 2 suggests that it reflects a promptly reversible deterioration in motor nerve function, such as increased conduction block.

The four patients with steroid induced motor deterioration all fitted the diagnostic category of demyelinating motor neuropathy, often associated with conduction block. ${ }^{9}$ Such patients are predominantly male, have slowly evolving multifocal muscle weakness usually worse in the arms, and normal sensation. Bulbar involvement has been recorded ${ }^{14}$ as in our patient 4. As in two of our patients, GM1 ganglioside antibodies occur in $11 \%$ to $84 \%$ of such patients, depending on the clinical subgroup. ${ }^{19}$ Such antibodies, however, are not specific markers for pure motor demyelinating neuropathy as they also occur although less often in sensorimotor demyelinating neuropathies. ${ }^{10}$ None of these four patients had the paraesthesiae or demonstrable sensory loss so common in conventional CIDP. ${ }^{6720}$ Unlike the patients with CIDP, the SAPs were preserved in the patients with MFMN, except in the 75 year old (patient 4). Sural nerve biopsy revealed no evidence of active sensory nerve fibre demyelination in the four patients with $M F M N$, unlike the findings often reported in sensorimotor CIDP. ${ }^{136}$

Clear improvement in strength and motor nerve conduction occurred in our two patients with MFMN (1 and 2) who were treated with IVIg. Symptomatic improvement started at three and seven days respectively after the start of IVIg (five days at $0.4 \mathrm{~g} / \mathrm{kg} /$ day), and lasted up to 10 weeks; lower doses were less effective. Other recent reports have shown similar improvement in MFMN after treatment with IVIg. ${ }^{12-15}$ Blinded, controlled trials of treatment are unlikely to be feasible for MFMN and these reports of clear cut responsiveness provide an empirical basis for establishing IVIg as the most effective, reasonably safe, contemporary treatment in MFMN. After IVIg treatment in our patients the pronounced early increase in the amplitude of the biphasic distal compound muscle action potentials combined with the persistence of polyphasic proximal compound muscle action potentials (some of which became longer in duration) suggests that the main process affecting distal conduction was a reversal of conduction block. The subsequent increase in maximal motor conduction velocity in some nerves probably reflects the unblocking of faster conducting fibres. Certainly the prompt clinical response to IVIg, and the irreversible deterioration induced by steroids, would be hard to attribute to mechanisms other than changes in the degree of conduction block. We did not perform serial studies of antiganglioside titres during steroid treatment and cannot comment on whether clinical worsening correlated with raised antibody titres, possibly resulting from steroid induced lymphocytosis. It is difficult to attribute the prompt improvement with IVIg to a putative anti-idiotype antibody neutralising a patho- genic antibody that has already bound to nerve fibres and caused conduction block. One cannot exclude the possibility that the IVIg solution, or steroids, alter the levels of other factors such as cytokines, which may mediate conduction block.

Given that steroids may induce deterioration in purely motor demyelinating neuropathy, how should we treat this condition? Although cyclophosphamide seems to be effective $^{11}$ most physicians would baulk at long term use of such a toxic drug except in seriously disabled patients. Currently IVIg seems to be the most effective and least toxic treatment. It should be recognised, however, that many patients with pure motor demyelinating neuropathy are inconvenienced rather than profoundly disabled by their disease, particularly early on. Thus conservative treatment may be realistic in the early years of a patient's disease, before introducing regular IVIg infusions once significant disability has accrued. We recommend that each patient's response is monitored closely when IVIg is initially introduced, firstly to be sure of its effectiveness in that particular patient and secondly to identify suitable dosages and treatment intervals. It may prove misleading to try and assess the response to IVIg in patients who are already taking prednisolone or cyclophosphamide, and who may have a changing background of weakness.

Until the specific biological mechanisms underlying the various syndromes of acquired demyelinating neuropathy are defined, the validity of differentiating them on clinical and electrophysiological grounds will be debated. For instance, how do patients with sensorimotor multifocal conduction block $^{21}$ relate to those with conventional symmetric sensorimotor CIDP on one hand, and MFMN on the other? Given our present lack of absolute criteria for differentiating subgroups of acquired demyelinating neuropathy, a clinical classification is important so as to choose appropriate modes of treatment. It is clearly important to avoid inducing deterioration with steroids in the purely motor subgroup, and yet to offer this valuable mode of treatment to those with sensorimotor CIDP.

We are grateful to Mrs Anne Richardson for preparing the manuscript.

1 Dyck PJ, Lais AC, Ohta M, Bastron JA, Okazaki H, Groover RV. Chronic inflammatory polyradiculopathy. Mayo Clin Proc 1975;50:621-37.

2 Matthews WB, Howell DA, Hughes RC. Relapsing corticosteroid-dependent polyneuritis. $f$ Neurol Neurosurg Psychiatry 1970;33:330-7.

3 Prineas JW, McLeod JG. Chronic relapsing polyneuritis. I Neurol Sci 1976;27:427-58.

4 Thomas PK, Lascelles RG, Hallpike JF, Hewer RI Recurrent and chronic relapsing Guillain-Barré polyneuritis. Brain 1969;92:589-606.

5 Dyck PJ, O'Brien PC, Oviatt K, et al. Prednisone improves chronic inflammatory demyelinating polyradiculoneuropathy more than no treatment. Ann Neurol 1982; ropathy more $136-41$.

6 Barohn RJ, Kissel JT, Warmolts JR, Mendell JR. Chronic inflammatory demyelinating polyradiculopathy: clinical inflammatory demyelinating polyradiculopathy: clinical
characteristics, course, and recommendations for diagcharacteristics, course, and recommendations
nostic criteria. Arch Neurol 1989;46:878-84.

7 McCombe PA, Pollard JD, McLeod JG. Chronic inflammatory demyelinating polyradiculoneuropathy. A clinical and electrophysiological study of 92 cases. Brain 1987;110:1617-30.

8 Dyck PJ, Daube J, O'Brien P, et al. Plasma exchange in 
chronic inflammatory demyelinating polyradiculoneuropathy. N Engl f Med 1986;314:461-5.

9 Pestronk A, Chaudhry V, Feldman EL, et al. Lower motor neuron syndromes defined by patterns of weakness, nerve conduction abnormalities and high titres of antiglycolipid antibodies. Ann Neurol 1990;27:316-26.

10 Sadiq SA, Thomas FP, Kilidireas K, et al. The spectrum of neurologic disease associated with anti-GM1 antibodies. Neurology 1990;40:1067-72.

11 Feldman EL, Bromberg MB, Albers JW, Pestronk A. Immuno-suppression treatment in multifocal motor neuropathy. Ann Neurol 1991;30:397-401.

12 Charles N, Benoit P, Vial C, et al. Intravenous immunoglobulin treatment in multifocal motor neuropathy. Lancet 1992;340:182.

13 Chaudhry V, Corse AM, Cornblath DR, et al. Multifocal motor neuropathy: response to human immunoglobulin. Ann Neurol 1993;33:237-42.

14 Kaji R, Shibasaki H, Kimura J. Multifocal demyelinating motor neuropathy: cranial nerve involvement and motor neuropathy: cranial nerve involvement

15 Nobile-Orazio E, Meucci N, Barbieri S, et al. High-dose intravenous immunoglobulin therapy in multifocal motor neuropathy. Neurology 1993;43:537-44.

16 Ad Hoc Subcommittee of American Academy of Neurology AIDS Task Force. Research criteria for diagnosis of chronic inflammatory demyelinating polynosis of chronic inflammatory demy

17 Jacobs JM, Love S. Qualitative and quantitative morphology of human sural nerve at different ages. Brain 1985;108: 897-924.

18 Gregson NA, Jones D, Thomas PK, Willison HJ. Acute motor neuropathy with antibodies to GM1 ganglioside. f Neurol 1991;238:447-51.

19 Gregson NA, Jones D, Willison H. Antibodies agains GM1 and other gangliosides in patients with motor neurone syndromes. In: Clifford-Rose, ed. New evidence in MNDIALS research. London: Smith Gordon 1991.

20 Dalakas MC, Engel WK. Chronic relapsing (dysimmune) polyneuropathy: pathogenesis and treatment. Ann Neurol 1981;9(suppl): 134-43.

21 Lewis RA, Sumner AJ, Brown MJ, Asbury AK Multifocal demyelinating neuropathy with persistent conduction block. Neurology 1982;32:958-64. 